\title{
Cimentos biocerâmicos em endodontia: revisão de literatura
}

\author{
Bioceramic sealers in endodontics: a literature review
}

\author{
Naggila Fernanda Figueiredo Lima* \\ Priscilla Raquel Negreiros dos Santos* \\ Marlus da Silva Pedrosa*** \\ Maraisa Greggio Delboni ${ }^{* * * *}$
}

\section{Resumo}

Introdução: cimentos biocerâmicos têm se tornado populares em endodontia devido às suas várias vantagens. Objetivo: revisar a literatura atualmente disponível sobre as propriedades de cimentos biocerâmicos em endodontia. Metodologia: uma pesquisa bibliográfica exploratória foi realizada utilizando as bases de dados eletrônicas Public Medline (PubMed), Scopus, Embase, e Web of Science, empregando os termos de busca "Bioceramic" AND "Endodontic". Os critérios de inclusão foram artigos de pesquisas originais e ensaios clínicos publicados em português, espanhol e inglês. Não foram aplicados limites de data de publicação. Resultados: foram identificados 30 estudos que abordaram propriedades de cimentos biocerâmicos em endodontia, publicados em um período de sete anos (2011-2017). As propriedades e características vantajosas destacadas nos estudos foram: biocompatibilidade, citotoxicidade, bioatividade, radiopacidade, $\mathrm{pH}$ e extravasamento (extravasamento não se enquadra como propriedade), adaptação marginal, resistência de união, resistência à fratura das raízes, capacidade seladora e propriedades antibacterianas. A alteração de cor também foi avaliada em alguns estudos. Conclusão: os estudos selecionados para esta revisão de literatura mostram que os cimentos endodônticos biocerâmicos apresentam propriedades promissoras para serem utilizados no tratamento de canais radiculares.

Palavras-chave: Cimentos dentários. Materiais biocompatíveis. Endodontia.

\section{Introdução}

As biocerâmicas são compostos cerâmicos biocompatíveis obtidos por vários processos químicos. Exibem excelentes propriedades de biocompatibilidade devido à sua similaridade com o processo biológico de formação de hidroxiapatita e à capacidade de induzir uma resposta regenerativa no corpo humano. As biocerâmicas apresentam capacidade osteoindutiva intrínseca, pois absorvem substâncias osteoindutoras na presença de processo de cicatrização óssea ${ }^{1}$.

Com o advento da nanotecnologia, tornou-se possível usar biocerâmica como cimento radicular agregando todos os benefícios das biocerâmicas ${ }^{2}$. Assim, sendo aplicáveis para uso odontológico e possuindo propriedades hidrofílicas, os cimentos biocerâmicos estão sendo introduzidos no mercado. A sua composição inclui silicatos tricálcicos e dicálcicos, fosfatos de cálcio, hidróxido de cálcio e óxido de zircónio como um radiopacificador ${ }^{3-5}$.

Cimentos biocerâmicos estão se tornando populares em endodontia como material de reparação de perfurações nas raízes e cimento obturador de canal radicular devido às suas propriedades, tais como: biocompatibilidade, $\mathrm{pH}$ elevado, não reabsorção, facilidade de manuseio no interior dos canais radiculares, aumento da resistência radicular, baixa citotoxicidade, além de não sofrerem contração e serem quimicamente estáveis ${ }^{6-8}$. 
Os cimentos biocerâmicos que foram recentemente utilizados no tratamento endodôntico apresentam uma literatura restrita sobre suas propriedades físicas e químicas ${ }^{1}$. Neste sentido, o presente trabalho, objetivou revisar a literatura atualmente disponível sobre as propriedades de cimentos biocerâmicos em endodontia.

\section{Métodos}

\section{Identificação e seleção de estudos relevantes}

Para o propósito deste estudo, seguimos as instruções fornecidas por Moher et al. ${ }^{9}$ (2009), em Preferred reporting items for systematic reviews and meta-analyses: the PRISMA statement. No intuito de responder a seguinte questão norteadora: "O que os estudos científicos mostram acerca das propriedades de cimentos biocerâmicos em endodontia?", uma pesquisa bibliográfica exploratória foi realizada, utilizando as bases de dados eletrônicas: Public Medline (PubMed), Embase, Web of Science e Scopus. Dois autores realizaram a pesquisa, empregando os termos de busca descritos na Tabela 1. Os critérios de inclusão foram: artigos de pesquisas originais e ensaios clínicos publicados em português, espanhol e inglês, sem limites de data de publicação.

Tabela 1 - Estratégia de busca para bases de dados adotadas

\begin{tabular}{l|l}
\hline Combinações de termos de busca & Base de dados \\
\hline $\begin{array}{l}\text { 1. bioceramic[All Fields] AND } \\
\text { endodontic[All Fields] }\end{array}$ & PubMed \\
$\begin{array}{l}\text { 2. TITLE-ABS-KEY ( bioceramic AND } \\
\text { endodontic) }\end{array}$ & Embase \\
$\begin{array}{l}\text { 3. bioceramic AND endodontic } \\
\text { 4. TITLE-ABS-KEY ( bioceramic AND } \\
\text { endodontic) }\end{array}$ & Web of Science \\
\hline
\end{tabular}

Fonte: dos autores.

Os estudos foram identificados e foram removidas as duplicações. Subsequentemente, títulos e resumos foram selecionados por relevância, considerando os critérios de exclusão. Em seguida, os demais estudos foram obtidos na íntegra e foram selecionados com os mesmos critérios, sendo os elegíveis incluídos nesta revisão.

Foram removidos estudos de revisão, manuscritos em outros idiomas não selecionados para este estudo, artigos não disponíveis em texto completo e publicações não relevantes/condizentes à temática proposta. Os estudos elegíveis também tiveram as suas listas de referências pesquisadas. Com base nos achados dos estudos, foram determinados os seguintes temas para análise crítica: ca- racterísticas dos estudos e materiais biocerâmicos em endodontia.

\section{Resulltados}

Para esta revisão, a pesquisa inicial resultou em um total de 117 títulos encontrados na busca eletrônica nas bases de dados: PubMed $(\mathrm{n}=30)$, Scopus ( $\mathrm{n}=37)$, Embase $(\mathrm{n}=21)$ e Web of Science $(n=29)$. Foram excluídos 59 estudos por duplicação, e 58 trabalhos foram selecionados como potencialmente relevantes para este estudo. Após a leitura de títulos e resumos, 19 trabalhos foram excluídos. Em seguida, os 39 estudos restantes foram obtidos na íntegra e avaliados quanto à elegibilidade, em consideração às propriedades de cimentos biocerâmicos em endodontia. Os resultados do estudo $(n=30)$ serão apresentados de forma descritiva em tópicos. Nenhum estudo de listas de referências foi adicionado (Figura 1).

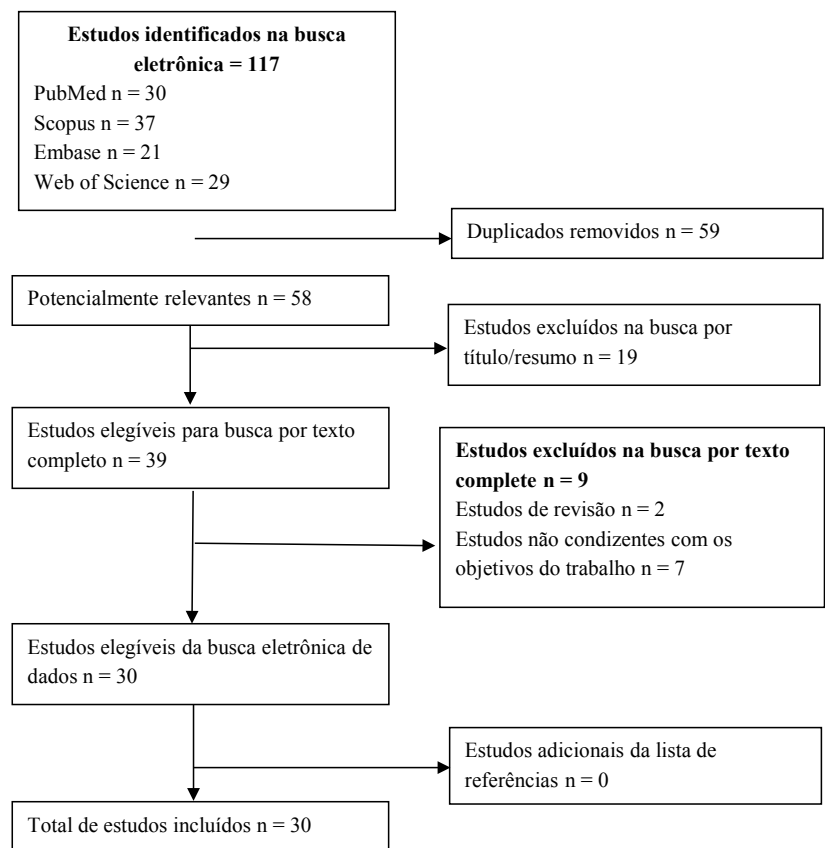

Figura 1 - Fluxograma para revisão dos artigos

Fonte: adaptado de Moher et al. ${ }^{9}$ (2009).

\section{Características dos estudos}

Foram identificados 30 estudos que se enquadraram nos critérios de inclusão mencionados na metodologia. Segundo nossos resultados, identificou-se que os estudos foram publicados em um período de sete anos (01/01/2011 - 06/10/2017). A cronologia dos resultados mostrou um interesse crescente em relação à temática bem como a necessidade de pesquisas futuras. Para melhor compreensão, os resultados acerca das propriedades dos cimentos biocerâmicos em endodontia são apresentados de forma categórica. 


\section{Biocompatibilidade e citotoxicidade}

Quando comparados em relação à sua citotoxicidade, o EndoSequence Root Repair Material (ERRM) e o Agregado Trióxido Mineral (MTA) exibem níveis semelhantes mínimos de citotoxicidade, bem como expressões de citocinas (IL-1b, IL-6, and IL-8 $)^{10}$. Comparando a citotoxicidade de dois materiais à base de biocerâmica, BioAggregate e iRoot (Innovative Bioceramix, IBC, Vancouver, Canada), em células MRC-5 de fibroblasto humano, Fayyad ${ }^{2}$ (2011) encontrou que ambos apresentaram uma biocompatibilidade aceitável e que o efeito citotóxico dos materiais foi dependente da concentração.

Com relação à biocompatibilidade in vitro do iRoot BP Plus (iRoot) comparada com White ProRoot MTA (MTA), iRoot mostrou-se biocompatível e ambos não induziram efeitos citotóxicos críticos, mesmo promovendo uma viabilidade significativamente menor que o MTA após 48 horas de exposição; iRoot não induziu efeitos citotóxicos críticos, porque a viabilidade celular permaneceu superior a $70 \%$ do grupo controle na maioria dos testes realizados ${ }^{11}$.

Para Willershausen et al. ${ }^{12}$ (2013), os materiais biocerâmicos como ERRM, ProRoot MTA e MTA-Angelus são biocompatíveis, e os autores sugerem esses materiais como obturadores retrógrados na cirurgia paraendodôntica, uma vez que sua adequação para uso clínico tenha sido demonstrada.

Comparando as características do cimento endodôntico biocerâmico EndoSequence BC sealer com AH Plus ${ }^{13}$, o cimento biocerâmico apresentou menor citotoxicidade e genotoxicidade em comparação ao AH Plus.

Estudos adicionais devem ser realizados para avaliar os componentes de subprodutos produzidos durante a presa do material, a fim de avaliar com mais precisão a citotoxicidade do EndoSequence BC Sealer. É possível, então, estabelecer uma correlação entre o tempo de presa do material e o grau de citotoxicidade $^{14}$.

\section{Bioatividade}

Shokouhinejad et al. ${ }^{15}$ (2012) avaliaram a bioatividade do Bioagregado (BA), EndoSequence Root Repair Material (ERRM) e branco ProRoot Mineral trióxido agregado (MTA), constatando que a exposição de MTA, BA e ERRM ao fluido de tecido simulado, PBS, resultou na precipitação de cristais de apatita, que se tornaram maiores com o aumento dos tempos de imersão. Pode-se concluir que todos os materiais biocerâmicos neste estudo são bioativos.

Güven et al. ${ }^{16}$ (2013) compararam o efeito do Agregado Mineral Trióxido (MTA) e iRoot SP, um cimento biocerâmico de canal radicular sobre a viabilidade celular, capacidade de deposição de tecido duro e diferenciação odontogênica de células-tronco germinativas humanas (hTGSCs). MTA e iRoot SP induziram a diferenciação de hTGSC em células semelhantes a odontoblastos, mas o MTA poderia proporcionar mais potencial indutivo e deposição de tecido duro em comparação com iRoot SP.

Chen et al. ${ }^{17}$ (2015) compararam a cicatrização após a cirurgia radicular utilizando o agregado de trióxido de mineral (MTA) e o EndoSequence Root Repair Material (ERRM) como material de obturação radicular em modelo animal. Como o MTA, o RRM é um material biocompatível com boa capacidade seladora. Contudo, no modelo animal, ERRM obteve uma melhor resposta de cicatrização do tecido adjacente. A tendência de cicatrização superior associada ao ERRM foi detectada pela tomografia computadorizada de feixe cônico (CBCT).

Carvalho et al..$^{18}$ (2017) avaliaram a bioatividade do EndoSequence Bioceramic Sealer e sua resistência de união à dentina (pelo teste micro push-out) em comparação com o cimento AH-Plus, verificando que o EndoSequence Bioceramic Sealer mostrou indicações de bioatividade e menor resistência de união à dentina.

\section{Alteração de cor}

Os materiais à base de biocerâmica Biodentine (Septodont, Saint-Maur-des-Fosses, França), ERRM, EndoSequence, ERRM putty (Brasseler, Savannah, GA), RMF, EndoSequence ERRM fast set paste (Brasseler) e $\mathrm{AH}+, \mathrm{AH}$ Plus sealer (Dentsply), não induzem alteração de cor perceptível na estrutura dentária quando deixados na câmara pulpar por períodos de tempo de seis meses. No entanto, mais estudos clínicos são necessários para confirmar esses achados ${ }^{19}$.

Ao comparar o potencial de descoloração do Endosequence Bioceramic Root Repair Material Fast Set Putty (ERRM) e do ProRootMTA (PMTA), quando colocados coronalmente em dentes humanos extraídos durante um período de quatro meses, dentes em tratamento com PMTA exibiram descoloração progressiva, enquanto aqueles com ERRM mantiveram a estabilidade de cor $^{20}$.

\section{Radiopacidade, pH e extravasamento}

Um único estudo ${ }^{7}$ visou investigar radiopacidade, $\mathrm{pH}$ e extravasamento de um cimento endodôntico biocerâmico (Endosequence BC Sealer), o qual apresentou radiopacidade significativamente menor do que o AH Plus. O Endosequence BC Sealer apresentou radiopacidade e extravasamento de acordo com as recomendações da ISO 6876/2001. A análise de $\mathrm{pH}$ mostrou que Endosequence BC Sealer apresentou pH e liberação de $\mathrm{Ca} 2$ + maior do que o AH Plus. As propriedades físico-químicas analisadas demonstram valores favoráveis para um cimento de canal radicular. 


\section{Adaptação marginal}

A adaptação marginal do EndoSequence Root Repair Material (ERRM) foi comparável à do $\mathrm{MTA}^{21}$. Entretanto, os cimentos à base de biocerâmica exibiram mais regiões contendo gaps quando comparados com cimentos à base de resina, o AH Plus. Uma explicação plausível para presença de gaps interfaciais pode ter sido a técnica de compactação lateral realizada.

\section{Resistência de união (Push-out)}

A resistência de união do cimento biocerâmico EndoSequence BC Sealer foi igual à do $\mathrm{AH}$ Plus, com ou sem smear layer ${ }^{22}$. Entretanto, a presença de solução salina tamponada com fosfato (PBS) dentro dos canais radiculares aumentou a resistência de união do cimento EndoSequence BC/guta-percha após uma semana de avaliação. Não foi encontrada diferença entre a resistência adesiva de EndoSequence BC selante/guta-percha, na presença ou ausência de PBS nos canais radiculares após dois meses $^{15}$.

O ERRM (EndoSequence Root Repair Material) apresentou resistência adesiva significativamente maior para as paredes do canal radicular em comparação com MTA e BA (Bioagregado) ${ }^{23}$. Sobre a resistência de união aplicada nos diferentes terços do canal radicular, o cimento BC-Sealer provou ser o melhor material de adesão em todos os terços do canal radicular sendo significativamente mais notável no terço apical ${ }^{24}$. Segundo Madhuri et al. ${ }^{25}$ (2016), a resistência de união do selante biocerâmico é a mais elevada, seguida pela do cimento à base de resina. A mais baixa resistência de união foi observada pelo cimento à base de MTA.

A utilização de EDTA a 17\%, sozinho ou seguido por $5,25 \%$ de $\mathrm{NaOCl}$, CHX a $2 \%$ ou solução salina, resultou em resistência adesiva semelhante à do EndoSequence BC Sealer nas paredes dentinárias $^{16}$. A resistência adesiva dos cimentos de resina (AH-Plus e Adseal) não é afetada pela solução de irrigação (hipoclorito de sódio ou clorexidina); contudo, a umidade do canal afeta negativamente a resistência de união do AH-Plus. Clorexidina (CHX) reduziu a resistência de união do cimento EndoSequence $\mathrm{BC}^{26,27}$. Os remanescentes de hidróxido de cálcio tiveram um efeito negativo sobre a resistência de união do AH-26 e BC Sealer ${ }^{27}$.

\section{Retratamento dos canais radiculares}

Com relação ao retratamento de canais radiculares obturados com guta-percha e três cimentos endodônticos diferentes: iRoot SP (biocerâmico), MTA Fillapex (à base de MTA) e AH-26 (à base de resina epóxica), nenhum dos cimentos testados foi completamente removido dos canais radiculares utilizando o sistema ProTaper Universal Retreatment (PTR ${ }^{28}$.
Entretanto, resultados de Oltra et al. ${ }^{29}$ (2017) mostraram que o cimento EndoSequence BC Sealer apresentou significativamente mais resíduo do que o AH Plus. Esses dados corroboram os de Zuolo et al. ${ }^{30}$ (2016), nos quais cimentos biocerâmicos exibiram significativamente mais remanescentes nos canais e exigiram maior tempo no retratamento. No entanto, independente da técnica e do cimento utilizados, todos os materiais são passíveis de deixar remanescentes.

\section{Resistência à fratura das raízes}

Topçuoğ et al. ${ }^{31}$ (2013) avaliaram a resistência à fratura de dentes preenchidos com três cimentos endodônticos diferentes: biocerâmico (Endosequence BC sealer), Mineral trioxide aggregate-based sealer (Tech Biosealer Endo) e selador à base de resina epoxica (AH Plus Jet). Em contraste com Tech Biosealer Endo, o Endosequence BC e o AH Plus Jet aumentaram a resistência à fratura de pré-molares com raízes únicas preenchidas com o material.

\section{Capacidade seladora}

Os cimentos endodônticos biocerâmicos exibiram resultados de infiltração semelhantes ao $\mathrm{MTA}^{32}$. Bioceramic Root-end Repair (BCRR) é equivalente ao MTA em capacidade de vedação. Corroborando isso, Antunes et al. ${ }^{33}$ (2016) mostraram que o MTA e o BioCeramic Root Repair Material (BC-RRM) apresentaram capacidade de vedação semelhante.

\section{Propriedades antibacterianas}

Singh et al. ${ }^{34}$ (2016) avaliaram, in vitro, as propriedades antibacterianas dos selantes (cimentos) endodônticos contra Enterococcus faecalis e observaram que EndoSequence BC Sealer, biocerâmico, apresentou atividade antibacteriana semelhante ao MTA e melhor desempenho em comparação com cimentos à base de resina e óxido de zinco eugenol.

\section{Discussão}

Foram identificados 30 estudos que abordaram as propriedades de cimentos biocerâmicos em endodontia publicados em um período de sete anos. Os estudos abordaram a biocompatibilidade, citotoxicidade, bioatividade, radiopacidade, $\mathrm{pH}$, adaptação marginal, resistência de união, retratamento, resistência à fratura das raízes, capacidade seladora, e propriedades antibacterianas. Alteração de cor foi relatada em alguns estudos.

A maioria dos estudos buscou comparar as propriedades de cimentos endodônticos biocerâmicos com cimentos endodônticos convencionais e com o MTA, encontrando evidências consideráveis que 
apoiam esse material como padrão-ouro para vários procedimentos clínicos ${ }^{35-38}$.

Um material ideal utilizado para reparo endodôntico deve ser biocompatível, radiopaco, antibacteriano, dimensionalmente estável, fácil de manipular, além disso, não deve ser afetado pela contaminação sanguínea ${ }^{10}$. As propriedades físico-químicas de liberação de $\mathrm{Ca} 2+, \mathrm{pH}$, e radiopacidade conferem aos cimentos biocerâmicos características favoráveis para emprego em endodontia ${ }^{7}$. Materiais biocerâmicos apresentam boas propriedades seladoras (vedamento) ${ }^{32,33}$, sendo capazes de aumentar a resistência dos dentes obturados ${ }^{31}$. Quando submetidos ao retratamento de canal radicular, esses materiais apresentam significativamente mais resíduos $^{29}$, exigindo maior tempo para o procedimento clínico ${ }^{30}$, o que pode ser explicado por sua maior adesão aos canais radiculares.

A biocompatibilidade dos materiais utilizados no tratamento endodôntico é de grande importância, porque podem entrar em contato com os tecidos perirradiculares e existe o risco de uma possível toxicidade sistêmica ${ }^{13}$. Os materiais EndoSequence Root Repair Material (ERRM) ${ }^{10,13}$, BioAggregate e iRoot $^{11}$, ProRoot MTA and MTA-Angelus ${ }^{13}$ e Endosequence $\mathrm{BC}$ sealer ${ }^{14,18}$ apresentaram biocompatibilidade aceitável, não induzindo efeitos citotóxicos críticos $^{12}$.

Um material bioativo é capaz de interagir com tecidos vivos, resultando na formação de uma camada de apatita, biomineralização, na interface material-tecido ${ }^{39,40}$. Assim, a avaliação da formação de apatita na superfície de um material, quando exposta a um fluido corporal simulado, é um método aprovado para examinar a bioatividade in vivo dos tecidos duros ${ }^{40}$. A bioatividade de materiais biocerâmicos endodônticos foi confirmada no Bioagrega$\mathrm{do}^{12}$, EndoSequence Root Repair Material ${ }^{12,18}$, ProRoo tMTA ${ }^{12}$, iRoot $\mathrm{SP}^{17}$.

Com relação à adaptação marginal, essa propriedade foi considerada aceitável para materiais biocerâmicos ${ }^{21,22}$, com elevada resistência adesiva nas paredes do canal radicular ${ }^{24,25}$, as quais sofrem pouca influência dos métodos de irrigação $0^{16}$. Entretanto, destaca-se que a resistência adesiva desses materiais pode sofrer alteração pela clorexidina ${ }^{26,27}$ e remanescentes de hidróxido de cálcio ${ }^{27}$, sendo necessária uma limpeza efetiva do canal radicular.

A descoloração dentária induzida por cimentos endodônticos é um achado comum que prejudica o resultado estético do tratamento endodôntico ${ }^{41}$. Segundo Ahmed et al. ${ }^{42}$ (2012), todos os cimentos endodônticos causam a descoloração do dente quando deixados na câmara pulpar. Essa descoloração é consequência de compostos dos cimentos que se espalham em túbulos dentinários durante ou após sua adaptação ${ }^{43}$. Evidências suportam que materiais biocerâmicos poderiam induzir menor escurecimento da estrutura dentária ${ }^{19,20}$.
A utilização de materiais com atividade antimicrobiana é considerada vantajosa no esforço para reduzir o número de microrganismos remanescentes, prevenir a infecção recorrente do canal radicular e ajudar na cicatrização de tecidos periapicais ${ }^{4}$. O EndoSequence BC Sealer, material biocerâmico, apresentou atividade bacteriana aceitável ${ }^{34}$.

\section{Conclusão}

Os estudos selecionados nesta revisão de literatura mostram que cimentos endodônticos biocerâmicos apresentam boas propriedades para uso no tratamento de canais radiculares. Entretanto, para fomentar sua aplicação clínica, observa-se a necessidade de mais estudos, in vivo, com metodologias acuradas no sentido de se obter dados mais confiáveis sobre suas propriedades.

\section{Abstract}

Introduction: bioceramic sealers are becoming popular in endodontics due to their several advantages. Objective: to review the literature currently available on the properties of bioceramic sealers in endodontics Methods: an exploratory bibliographic search was performed using the electronic databases Public Medline (PubMed), Scopus, Embase, and Web of Science, applying the keywords "Bioceramic" AND "Endodontic". Inclusion criteria were original research articles and clinical trials published in Portuguese, Spanish, and English. There were no limits for publication date. Results: 30 studies were identified for addressing the properties of bioceramic sealers in endodontics; they were published over a period of 7 years (2011-2017). The valuable properties and characteristics highlighted in the studies were biocompatibility, cytotoxicity, bioactivity, radiopacity, $\mathrm{pH}$ and extravasation (extravasation is not considered a property), marginal adaptation, bond strength, root fracture strength, sealing ability, and antibacterial properties. Some studies also assessed color change. Conclusion: the studies selected for this review of the literature show that bioceramic endodontic sealers have promising properties for root canal treatments.

Keywords: Dental sealers. Biocompatible materials. Endodontic.

\section{Referências}

1. Cheng L, Ye F, Yang R, Lu X, Shi Y, Li L, et al. Osteoinduction of hydroxyapatite/beta-tricalcium phosphate bioceramics in mice with a fractured fibula. Acta Biomater 2010; 6(4):1569-74.

2. Fayyad DM. Cytocompatibility of new bioceramic-based materials on human fibroblast cells (MRC-5). Oral Surg Oral Med Oral Pathol Oral Radiol Endod 2011; 112(6):e137-42.

3. Bueno CR, Valentim D, Marques VA, Gomes-Filho JE, Cintra LT, Jacinto RC, et al. Biocompatibility and biominera- 
lization assessment of bioceramic-, epoxy-, and calcium hydroxide-based sealers. Braz Oral Res 2016;30(1).

4. Koch K, Brave D. Bioceramic technology: the game changer in endodontics. Endodontic Practice US 2009; 12:7-11.

5. Zhang W, Li Z, Peng B. Assessment of a new root canal sealer's apical sealing ability. Oral Surg Oral Med Oral Pathol Oral Radiol Endod 2009; 107(6):e79-82.

6. Nasseh A. The rise of bioceramics. Endodontic Practice 2009 ; 2:17-22.

7. Candeiro GT, Moura-Netto C, D'Almeida-Couto RS, Azambuja-Júnior N, Marques MM, Cai S, et al. Cytotoxicity, genotoxicity and antibacterial effectiveness of a bioceramic endodontic sealer. Int Endod J 2015; 49(9):858-64.

8. Koch K. Bioceramic technologya game changer in endodontic obturation. NJAGD Wisdom 2009; 6:8-11.

9. Moher D, Liberati A, Tetzlaff J, Altman DG; Altman D, Antes G, et al. Preferred reporting items for systematic reviews and meta-analyses: the PRISMA statement. PLos Med 2009; 6(7):264-9.

10. Ciasca M, Aminoshariae A, Jin G, Montagnese T, Mickel A. A comparison of the cytotoxicity and proinflammatory cytokine production of EndoSequence root repair material and ProRoot mineral trioxide aggregate in human osteoblast cell culture using reverse-transcriptase polymerase chain reaction. J Endod 2012; 38(4):486-9.

11. De-Deus G, Canabarro A, Alves GG, Marins JR, Linhares ABR, Granjeiro JM. Cytocompatibility of the ready-to-use bioceramic putty repair cement iRoot BP Plus with primary human osteoblasts. Int Endod J 2012; 45(6):508-13.

12. Willershausen I, Wolf T, Kasaj A, Weyer V, Willershausen B, Marroquin BB. Influence of a bioceramic root end material and mineral trioxide aggregates on fibroblasts and osteoblasts. Arch Oral Biol 2013; 58(9):1232-7.

13. Candeiro GT, Moura-Netto C, D'Almeida-Couto RS, Azambuja-Júnior N, Marques MM, Cai S, et al. Cytotoxicity, genotoxicity and antibacterial effectiveness of a bioceramic endodontic sealer. Int Endod J 2015;49(9):858-864.

14. Loushine BA, Bryan TE, Looney SW, Gillen BM, Loushine RJ, Weller RN, et al. Setting properties and cytotoxicity evaluation of a premixed bioceramic root canal sealer.1. J Endod 2011; 37(5):673-7.

15. Shokouhinejad N, Hoseini A, Gorjestani H, Raoof M, Assadian H, Shamshiri AR. Effect of phosphate-buffered saline on push-out bond strength of a new Bioceramic sealer to root canal dentin. Dent Res J (Isfahan) 2012; 9(5):595.

16. Güven EP, Taşli PN, Yalvac ME, Sofiev N, Kayahan MB, Sahin F. In vitro comparison of induction capacity and biomineralization ability of mineral trioxide aggregate and a bioceramic root canal sealer. Int Endod J 2013; 46(12):1173-82.

17. Chen I, Karabucak B, Wang HG, Koyama E, Kohli MR, Nah $\mathrm{HD}$, et al. Healing after root-end microsurgery by using mineral trioxide aggregate and a new calcium silicate-based bioceramic material as root-end filling materials in dogs. $\mathrm{J}$ Endod 2015; 41(3):389-99.

18. Carvalho CN, Grazziotin-Soares R, Candeiro GTM, Martinez LG. Souza JP, Oliveira PS, et al. Micro Push-out Bond Strength and Bioactivity Analysis of a Bioceramic Root Canal Sealer. Iran Endod J 2017; 12(3):343-8.

19. Kohli MR, Yamaguchi M, Setzer FC, Karabucak B. Spectrophotometric Analysis of Coronal Tooth Discoloration Induced by Various Bioceramic Cements and Other Endodontic Materials. J Endod 2015; 41(11):1862-6.

20. Alsubait $\mathrm{S}$ et al. A Comparison of the Discoloration Potential for EndoSequence Bioceramic Root Repair Material
Fast Set Putty and ProRoot MTA in Human Teeth: an in vitro study. J Esthet Restor Dent 2016; 29(1):59-67.

21. Shokouhinejad N, Nekoofar MH, Ashoftehyazdi K, Zahraee $\mathrm{S}$, Khoshkhounejad M. Marginal adaptation of new bioceramic materials and mineral trioxide aggregate: a scanning electron microscopy study. Iran Endod J 2014; 9(2):144-8.

22. Shokouhinejad N, Gorjestani H, Nasseh AA, Hoseini A, Mohammadi M, Shamshiri AR. Push-out bond strength of gutta-percha with a new bioceramic sealer in the presence or absence of smear layer. Aust Endod J 2013; 39(3):102-6.

23. Shokouhinejad N, Hoseini A, Gorjestani H, Shamshiri AR. The effect of different irrigation protocols for smear layer removal on bond strength of a new bioceramic sealer. Iran Endod J 2013; 8(1):10-3.

24. Varguez AC, Basoco BIS, Vizcarra BG, Gomez LJV, Fernández DJ, Aguilera NR, et al. Comparative in vitro Study of the Bond Strength on Dentin of Two Sealing Cements: BC-SEALER and AH-PLUS. Rev. mex. ing. bioméd. 2016;37(2):115-122.

25. Madhuri GV, Varri S, Bolla N, Mandava P, Akkala LS, Shaik J. Comparison of bond strength of different endodontic sealers to root dentin: an in vitro push-out test. J Conserv Dent 2016; 19(5):461-4.

26. Razmi H, Bolhari B, Karamzadeh Dashti N, Fazlyab M. The Effect of Canal Dryness on Bond Strength of Bioceramic and Epoxy-resin Sealers after Irrigation with Sodium Hypochlorite or Chlorhexidine. Iran Endod J 2016; 11(2):129-33.

27. Ghabraei S, Bolhari B, Yaghoobnejad F, Meraji N. Effect of intra-canal calcium hydroxide remnants on the push-out bond strength of two endodontic sealers. Iran Endod J 2017; 12(2):168-72.

28. Uzunoglu E, Yilmaz Z, Sungur DD, Altundasar E. Retreatability of Root Canals Obturated Using Gutta-Percha with Bioceramic, MTA and Resin-Based Sealers. Iran Endod J 2015; 10(2):93-8.

29. Oltra E, Cox TC, LaCourse MR, Johnson JD, Paranjpe A. Retreatability of two endodontic sealers, EndoSequence BC Sealer and AH Plus: a micro-computed tomographic comparison. Restor Dent Endod 2017; 42(1):19-26.

30. Zuolo AS, Zuolo ML, da Silveira Bueno CE, Chu R, Cunha RS. Evaluation of the Efficacy of TRUShape and Reciproc File Systems in the Removal of Root Filling Material: an ex vivo micro-computed tomographic study. J Endod 2016; 42(2):315-9.

31. Topçuoğlu HS, Tuncay Ö, Karataş E, Arslan H, Yeter K. In vitro fracture resistance of roots obturated with epoxy resin-based, mineral trioxide aggregate-based, and bioceramic root canal sealers. J Endod 2013; 39(12):1630-3.

32. Leal F, De-Deus G, Brandão C, Luna AS, Fidel SR, Souza EM. Comparison of the root-end seal provided by bioceramic repair cements and White MTA. Int Endod J 2011; 44(7):662-8.

33. Antunes HS, Gominho LF, Andrade-Junior CV, Dessaune-Neto N, Alves FR, Rôças IN, et al. Sealing ability of two root-end filling materials in a bacterial nutrient leakage model. Int Endod J 2016; 49(10):960-5.

34. Singh G, Gupta I, Elshamy FM, Boreak N, Homeida HE. In vitro comparison of antibacterial properties of bioceramic-based sealer, resin-based sealer and zinc oxide eugenol based sealer and two mineral trioxide aggregates. Eur J Dent 2016; 10(3):366-9.

35. Mangin C, Yesilsoy C, Nissan R, Stevens R. The comparative sealing ability of hydroxyapatite cement, mineral trioxide aggregate, and super ethoxybenzoic acid as root-end filling materials. J Endod 2003; 29:261-4. 
36. Bortoluzzi EA, Broon NJ, Bramante CM, Consolaro A, Garcia RB, de Moraes IG, et al. Mineral Trioxide Aggregate with or without Calcium Chloride in Pulpotomy. J Endod 2008;34(2):172-5.

37. Min KS, Park HJ, Lee SK, Park SH, Hong CU, Kim HW, et al.Effect of mineral trioxide aggregate on dentin bridge formation and expression of dentin sialoprotein and heme oxygenase-1 in human dental pulp. J Endod 2008;34(6):666-70

38. Sarris S, Tahmassebi JF, Duggal MS, Cross IA. A clinical evaluation of mineral trioxide aggregate for root-end closure of non-vital immature permanent incisors in children-a pilot study. Dental Traumatology 2008; 24:79-85.

39. Hench LL, Wilson J. Surface-active biomaterials. Science 1984; 226:630-6.

40. Kokubo T, Takadama H. How useful is SBF in predicting in vivo bone bioactivity? Biomaterials 2006; 27:2907-15.

41. Tour Savadkouhi S, Fazlyab M. Discoloration Potential of Endodontic Sealers: a brief review. Iran Endod J 2016; $11(4): 250-4$.

42. Ahmed HM, Abbott PV. Discolouration potential of endodontic procedures and materials: a review. Int Endod J 2012; 45(10):883-97.

43. Parsons JR, Walton RE, Ricks-Williamson L. In vitro longitudinal assessment of coronal discoloration from endodontic sealers. J Endod 2001; 27(11):699-702.

\section{Endereço para correspondência:}

Marlus da Silva Pedrosa

Rua Senador Joaquim Pires, 723, Ininga

CEP 64049-590 Teresina, Piauí, Brasil

Telefone: (86) 99492-4142

E-mail: marluspedrosa@gmail.com

Recebido: 03/07/2017. Aceito: 30/07/2017. 